\title{
Is there a relationship between epicardial fat tissue thickness and Tp-Te/QT ratio in healthy individuals?
}

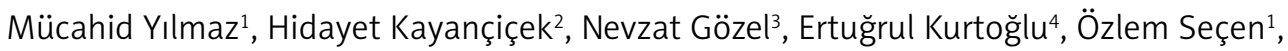

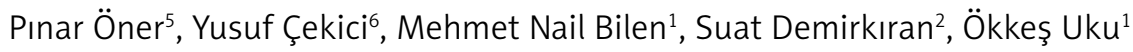

'Department of Cardiology, Elazı ğ Education and Research Hospital, Elazığ, Turkey ${ }^{2}$ Department of Cardiology, Elazığ Medical Park Hospital (Affiliated with Istinye University), Elazığ, Turkey

${ }^{3}$ Department of Internal Medicine, Firat University School of Medicine, Elazığ, Turkey ${ }^{4}$ Department of Cardiology, Malatya Education and Research Hospital, Malatya, Turkey ${ }^{5}$ Department of Microbiology, Elazığ Education and Research Hospital, Elazığ, Turkey ${ }^{6}$ Department of Cardiology, Dr. Ersin Arslan Education and Research Hospital, Gaziantep, Turkey

Submitted: 7 September 2019 Accepted: 10 April 2020

Arch Med Sci Atheroscler Dis 2020; 5: e127-e139

DOI: https://doi.org/10.5114/aoms.2020.96147

Copyright $\odot 2020$ Termedia \& Banach

\section{Abstract}

Introduction: Epicardial fat is a tissue that releases many proinflammatory and atherogenic mediators, with endocrine and paracrine effects on the heart. In this study, the implication of the EFT thickness (EFTt) on transmural dispersion of repolarisation (TDR) was analysed utilizing the T-wave peak to end interval (Tp-Te), the Tp-Te dispersion (Tp-Te (d)), and the Tp-Te/QT ratio. Material and methods: One thousand seven hundred and thirteen subjects were enrolled in the research. The subjects were chosen to be healthy individuals, without any cardiovascular/systemic disorders or risk factors for atherosclerosis. Transthoracic echocardiography (TTE) was applied to all subjects, and EFTt was measured in both diastole and systole. The ECG measurements were taken from standard 12-lead surface ECG.

Results: Correlation analysis revealed that the EFTt is highly associated with the Tp-Te interval, Tp-Te/QT ratio, Tp-Te (d), increasing age, body mass index (BMI), body surface area (BSA), left ventricular (LV) mass, LV mass index, plasma glucose during fasting, triglycerides, and low-density lipoprotein cholesterol.

Conclusions: The study results showed that increased EFTt was associated with increased TDR values of Tp-Te, Tp-Te (d), and Tp-Te/QT ratio, even in the absence of other factors that could increase TDR and EFTt. Therefore, it can be stated that increased EFTt may cause an increase the risk for ventricular arrhythmia.

Key words: fat tissue of epicardium, repolarisation inhomogeneity of ventricle, predisposition to ventricular arrhythmia.

\section{Introduction}

Epicardial fat tissue (EFT) resides between the visceral pericardium and myocardium, which is a unique fat compartment that shares a similar embryological origin with the visceral fat depot. The EFT is known to have many important cardiac effects and roles. Namely, the complex physiopathology of coronary atherosclerosis corporates with the local cardiac effects of the EFT [1]. EFT is an organ that excretes many proinflammatory
Corresponding author:

Dr. Mücahid Yılmaz Department of Cardiology Elazığ Education and Research Hospital Elazığ, Turkey Phone: +90 5337719783 E-mail: mucahid.yilmaz@ hotmail.com 
and atherogenic mediator factors with endocrine and paracrine effects on the heart. These mediators may cause cardiovascular disease, leading to a change in the metabolism of arterial endothelial/ smooth muscle cells and heart cells [2].

In recent studies, it was stated that the Tp-Te interval was an indicator of the transmural dispersion of repolarisation (TDR) (apicobasal, transmural, and global). The interval value of the Tp-Te can be identified as the distance between the highest value ( $T$ peak) and the endpoint ( $T$ end) of the $T$ wave, which is derived from ECG $[3,4]$. However, there is need to identify whether it is effected by body weight and heart variation issues [5]. Recently it has been claimed that the Tp-Te/QT ratio is not altered by fluctuations of the heart rate and that it is more reliable in demonstrating predisposition to ventricular arrhythmia than other known calculations [5, 6].

EFT mainly covers all coronary vessels. Also it surrounds the outer wall of the right ventricle and the left ventricular apex; hence, the EFT is known to have an influential effect on human cardiac conduction $[7,8]$. The possible relation between EFT thickness (EFTt) and ventricular TDR was analysed. The interval of Tp-Te, dispersion of Tp-Te $\mathrm{Tp}-\mathrm{Te}$ (d), and the Tp-Te/QT ratio were used as simple, noninvasive indicators of ventricular repolarisation inhomogeneity.

There is only one study with low patient participation [9]. It was carried out in the absence of the tests (such as myocardial perfusion scintigraphy and exercise stress test) excluding the existence of myocardial ischaemia. As is known that in myocardial ischaemia the risks associated with atherosclerosis, i.e. diabetes mellitus (DM), hypertension $(\mathrm{HT})$, and smoking, in addition to structural heart diseases, give rise to an increase in ventricular repolarisation parameters (TDR) and the heterogeneity of ventricular repolarisation. When viewed from the aforementioned aspect, there currently has been no study that analysed the link between EFTt and ventricular repolarisation parameters directly. We aimed to investigate whether there is a direct connection between EFTt and potential ventricular arrhythmia predisposition with broad patient participation.

\section{Material and methods}

\section{Population of the study}

A total of 1713 consecutive subjects (893 female and 820 male), who applied to Elazig Education and Research Hospital cardiology outpatient clinics and met the inclusion criteria between December 2016 and June 2018, were included in the study (Figure 1). The ages of the subjects varied between 17 and 75 years, and they had neither any cardiovascular/systemic disorders nor risk fac- tors for atherosclerosis (except hyperlipidaemia), detected by transthoracic echocardiography (TTE), myocardial perfusion scintigraphy, or exercise stress test. The study was carried out in conformity with the principles of the Helsinki declaration, and ethical approval was taken from the Presidency of T.C. Firat University Ethics Committee.

Exclusion criteria were electrolyte imbalance, usage of drugs that might influence the QT interval (including diltiazem, propafenone, $\beta$-blockers, verapamil, amiodarone, probucol, terfenadine, erythromycin, clarithromycin, anti-depressant agent, anti-psychotic agent), pregnancy, and being a professional athlete. In addition, participants who had right or left bundle-branch block determined in 12lead surface ECG and participants who had missing data were not included in the analysis. Lastly, participants who met the criteria for the inclusion were selected consecutively to avoid selection bias.

The physical examinations and laboratory analysis were conducted to record fasting blood glucose and lipid levels, blood pressure, body mass index (BMI), and body surface area (BSA) parameters. Patients who were taking antihypertensive drugs were not included in the analysis. Also, blood pressure measurements were taken to identify hypertensive patients (i.e., according to blood pressure records, subjects with diastolic blood pressure $\geq 90 \mathrm{~mm} \mathrm{Hg}$ and systolic blood pressure higher than $\geq 140 \mathrm{~mm} \mathrm{Hg}$ were not accepted to be subjects of the study).

To prove that a subject was diabetic, usage of antidiabetic medications or a measurement of fasting blood glucose level $\geq 126 \mathrm{mg} / \mathrm{dl}$ was required, and these subjects were not included in analysis.

BMI and BSA were evaluated according the following formulas:

$$
\begin{gathered}
\text { BMI: (weight }(\mathrm{kg})) / \text { height }^{2}\left(\mathrm{~m}^{2}\right) \\
\text { and BSA (Mosteller formula): } \\
\sqrt{ }((\text { height })(\mathrm{cm}) \times \text { weight }(\mathrm{kg}) / 3600)
\end{gathered}
$$

\section{Electrocardiographic measurements}

By tuning the voltage value to $10 \mathrm{~mm} / \mathrm{mV}$ and the paper speed to $50 \mathrm{~mm} / \mathrm{s}$, broadly used ECG equipment (CardiofaxV model 9320, Nihon Kohden, Tokyo, Japan) was utilised to record the 12-lead ECG. After scanning the whole of the ECG recording, the intervals of the Tp-Te, QT, and $R R$ were evaluated. The evaluations were done with the help of a computer program coded in MATLAB $^{\circledR}$ (MathWorks, Natick, Massachusetts, U.S.A.) that written by an engineer. These codes were based on image manipulation principles.

The QT interval was determined by intersecting the start value of the QRS with the final value of the $T$ wave. This interval was identified by taking 


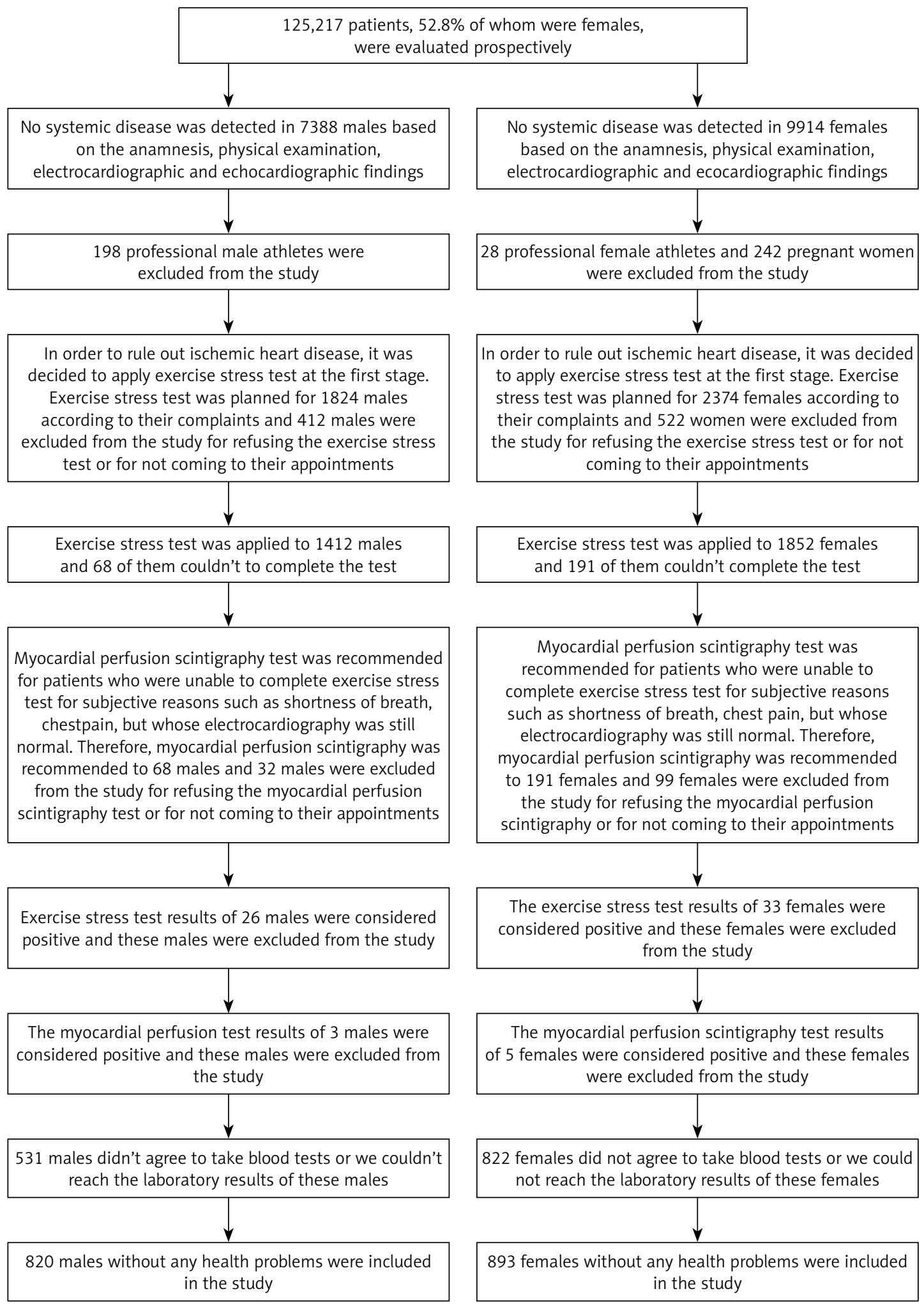

Figure 1. Subject inclusion flowchart diagram

into account the $T$ wave and the isoelectric line. The interception of the tangent (designed from the downward part of the T wave) and isoelectric line yielded the QT interval [10]. Such measurements were taken from $V_{1}-V_{6}$ derivations. The QTmax val-

ues were recorded by determining the maximum values, and the QTc was calculated by Bazett's formula (Figure 2) [11]. The interval starting from the highest point of the $T$ wave and ending at the end point of the T wave was defined as Tp-Te (Figure 3). 


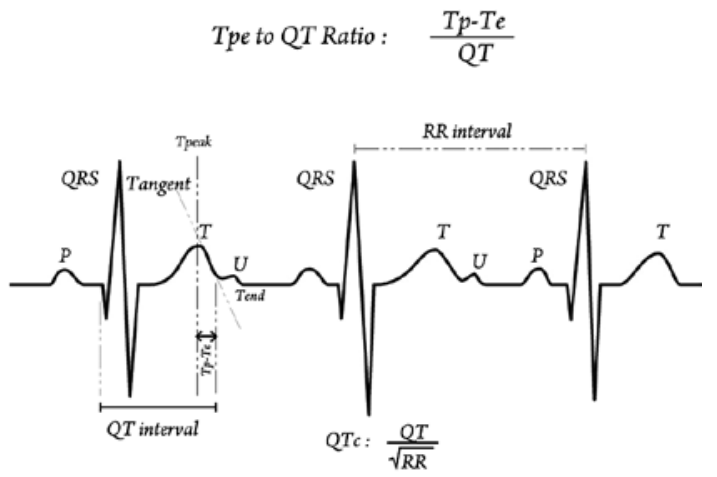

Figure 2. Bazett formula and Tp-Te/QT ratio

The final point of the T wave was identified as the point of interception of the tangent line and the isoelectric line (when an $U$ wave was not subsequent to the $T$ wave or if the $T$ wave was not distinct from the sequent $U$ wave). When the $T$ wave was followed by an $U$ wave, the lowest point between the $T$ and $U$ waves was determined as the final point of the T wave (Figure 1) [10]. The T peak was identified as the nadir of the $T$ wave when negative or biphasic $T$ waves were present (Figure 4) [12]. If a notched T wave was present, the final point of the QT distance was identified by utilising the tangent line drawn from the downward part of the second notch (Figure 5) [10].

During the calculations, only the T wave amplitudes greater than $1.5 \mathrm{~mm}$ were included in the measurements. Otherwise they were neglected. All of the precordial derivations were utilised for the Tp-Te interval measurements. The largest values were accepted as the Tp-Te interval. The TpTe (d) values were determined by subtracting the values corresponding to the maxima and minima

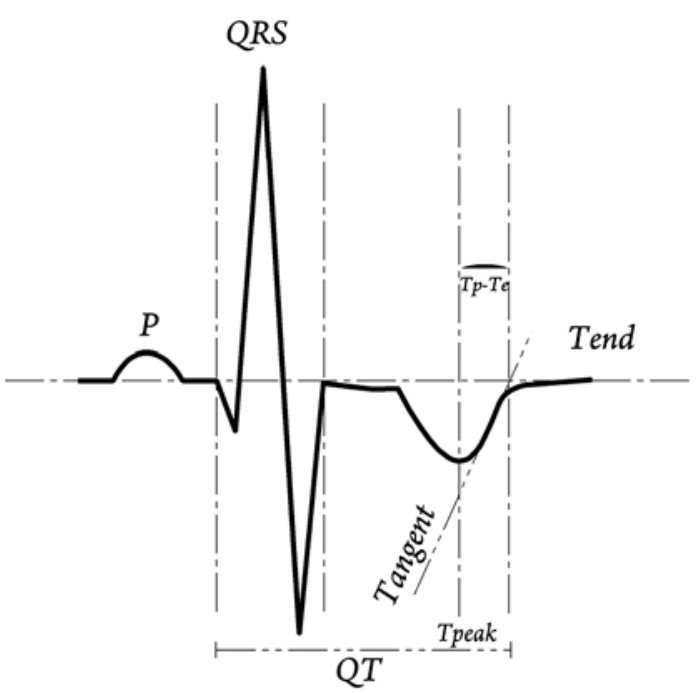

Figure 4. Schematic presentation of the measurement of the Tp-Te interval in the presence of a negative $T$ wave

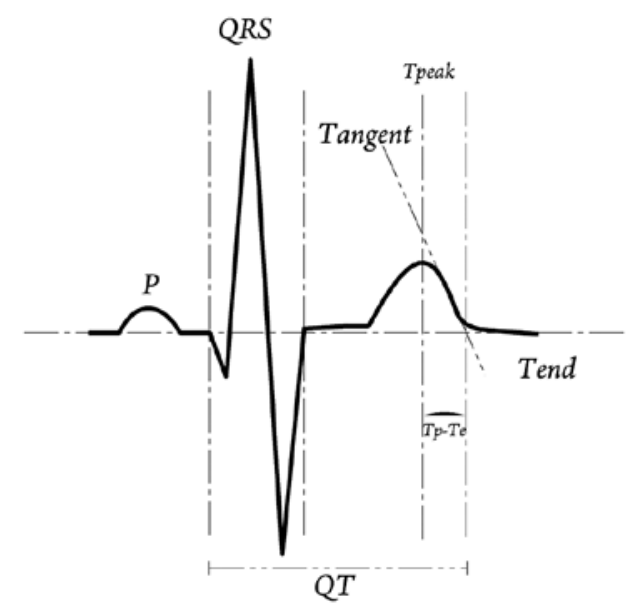

Figure 3. Schematic presentation of the measurement of the Tp-Te and QT interval

of the Tp-Te intervals, which resided from $V_{1}$ to $V_{6}$ derivations. The value of the Tp-Te/QT ratio was estimated utilising Tp-Te and QTmax values.

\section{Echocardiography}

In order to perform the transthoracic echocardiography, a Vivid 5 instrument with a $2.5 \mathrm{MHz}$ transducer (GE Medical Systems, Milwaukee, WI, USA) was applied. American Society of Echocardiography recommendations were followed [13]. Interventricular septum thickness, (IVS), posterior wall thickness (PW), and systolic and diastolic diameters of the left ventricle (LV) were measured with M-mode echocardiography. The ejection frac-

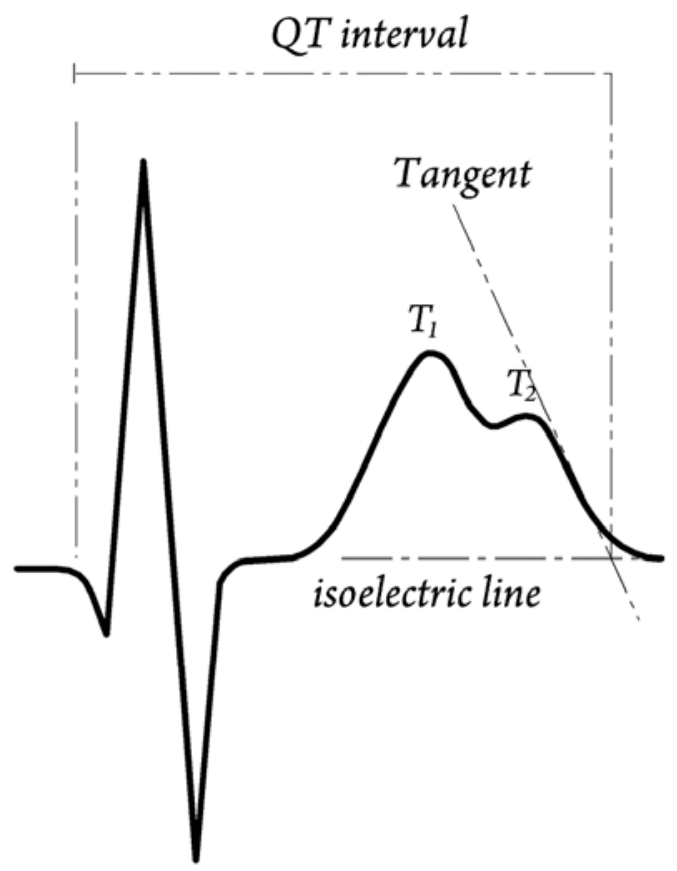

Figure 5. Maximum slope intercept method in the presence of a notched T wave 


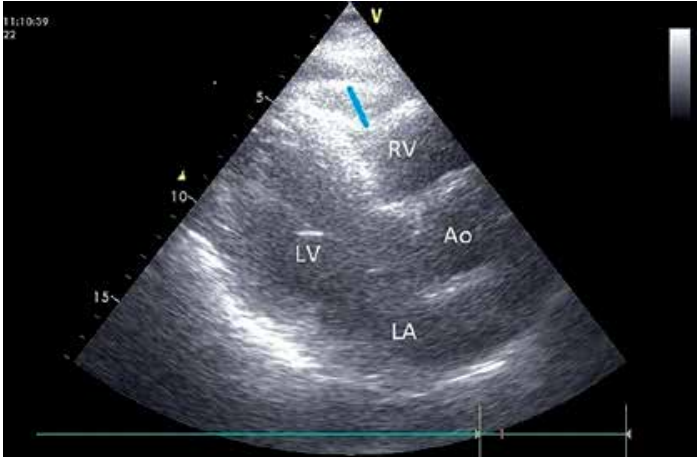

Figure 6. Measurement of epicardial fat tissue thickness (EFTt) by two-dimensional transthoracic echocardiography

Ao - aorta, LA - left atrium, $R V$ - right ventricle, $L V$ - left ventricle.

tion of the left ventricle was measured with the help of the Teichholz method [13]. The measurements of EFTt were carried out by a procedure suggested by lacobellis et al. [14]. In accordance with the aforementioned procedure, the measurements were taken on the outer wall of the right ventricle. The EFT was discriminated by determining the echo-free space between the pericardium visceral lamina and external wall of the myocardium (Figure 6). Evaluations on M-mode strips were gathered from longitudinal cursor beam orientation in three cardiac cycles at the end-systole and end-diastole. At any side of the figures, the maximum values were measured and the derived values were averaged. The method that was utilised to determine the LV mass is Deveureux's formula [15]. The formula is shown below: LV mass $[\mathrm{g}]=0.8 \times(1.04 \times((($ LVEDD + IVSd + PWd $)^{3}-\left(\right.$ LVEDD $\left.\left.\left.^{3}\right)\right)\right)+0.6$, where LVEDD is LV end-diastolic diameter.

LV mass index $\left(\mathrm{g} / \mathrm{m}^{2}\right)$ was computed with help of the following formula [15]: LV mass index [g/m²] $=$ left ventricular mass/BSA.

Respectively, LV end-diastolic volume (LVEDV) and LV end-systolic volume (LVESV) values were computed as follows [16]: LVEDV $[\mathrm{ml}]=(7 / 2.4+$ LVEDD $) \times \operatorname{LVEDD}^{3}, \operatorname{LVESV}[\mathrm{ml}]=(7 / 2.4+\operatorname{LVESD}) \times$ LVESD $^{3}$.

LVEDV index (LVEDVI) was computed with help the of the following formula [15]: LVEDVI $\left[\mathrm{ml} / \mathrm{m}^{2}\right]$ $=\mathrm{LVEDV} / \mathrm{BSA}$.

EF (LV ejection fraction) and FS (fractional shortening) were evaluated with the help of the following formulas, respectively [16]: EF (\%) = $(($ LVEDV - LVESV)/LVEDV) $\times 100$, FS $(\%)=(($ LVEDD - LVESD)/LVEDD) $\times 100$

\section{Exercise stress test}

In order to conduct the stress test, a Cardiosis TEPA Exercise Stress Test device (TEPA Medical and Electronic Products Industry and Trade Company, Ankara, Turkey) was utilised. Specifically, the tests were done according to the Bruce or modified Bruce treadmill protocols. Such protocols are known to be non-invasive for functional capacity and exercise tolerance for patients who are doubted to have cardiovascular disorders [17].

\section{Myocardial perfusion scintigraphy}

Myocardial perfusion scintigraphy test was conducted utilising the treadmill according to Bruce or modified Bruce protocols. Sestamibi (MIBI) tagged by $10 \mathrm{mCi} 99 \mathrm{mTc}$ (Cardio-Spect, Medi-Radiopharma, Budapest, Hungary) were applied intravenously (IV) into the subjects when the maximum HR (85-100\%) was reached. After $30 \mathrm{~min}$, the gated SPECT (single-photon emission computed tomography) imaging was taken. Imaging was carried out using a GE Infinia GP3 gamma monitor system (General Electric Healthcare, Tirat Carmel, Israel) with a low-energy, high-resolution (LEHR) collimator. Images were evaluated using Emory Cardiac Toolbox (ECTb) myocardial quantification software (General Electric Healthcare Company).

\section{Statistical analysis}

The statistical evaluations of the results of this study was done with the help of the SPSS 16.0 (SPSS Inc., Chicago, IL, USA) analysis program for Windows. With the exception of the Tp-Te/QTc ratio and BSA $\left(\mathrm{m}^{2}\right)$, all of the continuous values did not fit to the normal distribution, and the Kolmogorov-Smirnov test was used to evaluate these variables. Descriptive statistics test was used to evaluate the Tp-Te/QTc ratio and BSA $\left(\mathrm{m}^{2}\right)$. These values are shown as means with standard deviations. All other data were presented as medians with $25^{\text {th }}-75^{\text {th }}$ percentiles. Student's $t$-test and Mann-Whitney $U$ test were used to compare groups for continuous variables. Bonferroni correction was used for one-way ANOVA test to compare subgroups (BMI $<25 \mathrm{~kg} / \mathrm{m}^{2}, 25 \mathrm{~kg} / \mathrm{m}^{2}<\mathrm{BMI}$ $<30 \mathrm{~kg} / \mathrm{m}^{2}, 30 \mathrm{~kg} / \mathrm{m}^{2}<\mathrm{BMI}$ ). Degrees of association between continuous variables were analysed by Pearson's correlation analysis. Multivariate linear regression analysis was performed to determine which clinical variables were independently related to the Tp-Te interval and $\mathrm{Tp}-\mathrm{Te} / \mathrm{QT}$ ratio. The Tp-Te interval and Tp-Te/QT ratio were used in the model as dependent variables. EFTts, EFTtd, BMI $\left(\mathrm{kg} / \mathrm{m}^{2}\right)$, BSA $\left(\mathrm{m}^{2}\right.$, LV mass $(\mathrm{g})$, LV mass index $\left(\mathrm{g} / \mathrm{m}^{2}\right)$, LVEDV $(\mathrm{ml})$, LVEDV index $\left(\mathrm{ml} / \mathrm{m}^{2}\right)$, age, fasting plasma glucose $(\mathrm{mg} / \mathrm{dl})$, LDL-C (mg/dl), triglycerides $(\mathrm{mg} / \mathrm{dl})$, and $\mathrm{HDL}-\mathrm{C}(\mathrm{mg} / \mathrm{dl})$ were treated as independent variables. Results were presented as $\beta$ coefficients and $95 \%$ confidence 
intervals $(\mathrm{Cl}) . P<0.05$ was required for statistical significance.

\section{Results}

In this study, there were 1713 (820 males) healthy subjects, 1048 of whom were overweight $\left(25 \mathrm{~kg} / \mathrm{m}^{2}<\mathrm{BMI}<30 \mathrm{~kg} / \mathrm{m}^{2}\right)$ and 215 were obese $\left(30<\mathrm{BMI} \mathrm{kg} / \mathrm{m}^{2}\right)$. The median ages of all the study participants, males and females, were 43.0 (30.0-55.0), 41.0 (30.0-53.0), and 45.0 (31.0-55.0) years, respectively. The median Tp-Te interval, $\mathrm{Tp}-\mathrm{Te} / \mathrm{QT}$ ratio, and $\mathrm{Tp}-\mathrm{Te}(\mathrm{d})$ were 68.0 (64.0-70.0) ms, 0.18 (0.18-0.19), and 15.0 (10.020.0) $\mathrm{ms}$, and the mean TP-Te/QTc ratio was 0.17 \pm 0.015 , respectively. When analysing the same electrocardiographic parameters with respect to BMI $\left(\mathrm{BMI}<25 \mathrm{~kg} / \mathrm{m}^{2}, 25 \mathrm{~kg} / \mathrm{m}^{2}<\mathrm{BMI}<30 \mathrm{~kg} / \mathrm{m}^{2}\right.$, $\left.30 \mathrm{~kg} / \mathrm{m}^{2}<\mathrm{BMI}\right)$ the median Tp-Te interval was $63.0(60.0-66.0) \mathrm{ms}, 68.0(66.0-70.0)$, and 72.0 (68.0-75.0) $\mathrm{ms}$, respectively; the $\mathrm{Tp}-\mathrm{Te} / \mathrm{QT}$ ratio was 0.18 (0.17-0.18), $0.18(0.18-0.19)$, and 0.19 (0.18-0.20), respectively; the $\mathrm{Tp}-\mathrm{Te}(\mathrm{d})$ was 10.0 (10.0-15.0) ms, 16.0 (10.0-20.0), and 20.0 (12.025.0), respectively; and the mean TP-Te/QTc ratio was $0.16 \pm 0.014,0.17 \pm 0.014$, and $0.17 \pm 0.015$, respectively. Echocardiographic measurements, baseline clinical characteristics of the study participants, and findings for ventricular repolarisation parameters are summarised below (Tables I, II).

The median EFTtd (measured in diastole) was $2.5(1.8-3.2) \mathrm{mm}$ and median EFTts (measured in systole) was 5.0 (3.4-6.0) mm (Table I). Pearson's correlation analyses showed that EFTtd and EFTts were meaningfully related to: age of the subjects, plasma glucose of fasting, high density lipoprotein-cholesterol (HDL-C), low-density lipoprotein-cholesterol (LDL-C), triglycerides, Tp-Te, TPTe/QT ratio, TP-Te/QTc ratio, Tp-Te(d), BMI, BSA, LVEDV, LVEDV index, LV mass, and LV mass index (Table III, Figure 7).

Multivariate linear regression analysis results showed that Tp-Te interval and Tp-Te/QT ratio was independently related to age, BMI $\left(\mathrm{kg} / \mathrm{m}^{2}\right)$, LV mass (g), LVEDV $(\mathrm{ml})$, LVEDV index $\left(\mathrm{ml} / \mathrm{m}^{2}\right)$, and LDL-C. An independent relationship between EFTd and EFTs and Tp-Te and Tp-Te / QT ratio could not be detected (Tables IV, V).

\section{Discussion}

We analysed the relational association between TDR indexes (Tp-Te interval, Tp-Te(d), TpTe/QT ratio) and EFTt with the study conducted. The outcomes of the research revealed that there was a significant relationship between the aforementioned parameters. In addition, it was also observed that EFTt had a significant association with age, BMI, and hyperlipidaemia (Table III). On the other hand, an independent relationship between TDR and EFTt was not determined in the multivariate linear regression analysis (Tables IV, V).

Epicardial fat is a kind of visceral fat that deposits in the heart. EFT is situated between the visceral segment of pericardium and the myocardium. It is known to be highly active, with a fatty acid metabolism, and it bears highly expressed thermogenic genes [8]. The functional complexity of human epicardial fat is not fully elucidated. However, the role of epicardial fat in the heart can generally be identified as mechanical, metabolic, thermogenic, and endocrine/paracrine [18].

The EFT is very close to the myocardium, which means it plays a vital role. Anatomically, there is no border between the myocardium and the EFT, and because of this anatomical phenomenon the myocardium is effected by some metabolites that are released from the EFT. Specifically, some of the metabolites can be named as the adipokines and cytokines $[19,20]$. Recent studies suggest that the cytokines that are released from the EFT has a significant effect on some cardiovascular disease development [21-24].

EFT is located in the interventricular and atrioventricular grooves that cover the atria, the main branches of coronary arteries, the outer wall of the right ventricle, and the apex of the left ventricle [8]. The increase in the size of the epicardial fat causes the coronary arteries and myocardium to be surrounded by fat. It is also a known fact that the fat can get into the connective tissue out-setting the subepicardial connective tissue, which stands in the muscle bundles and muscle fibres [19]. Furthermore, when extreme obesity exists, the heart may become completely covered with fat that can be as thick as $20 \mathrm{~mm}$ [25]

Today, EFT has attracted the interest of many researchers owing to its anatomical and functional characteristics, which mainly stem from its closeness to the myocardium. Many research studies have been conducted to illuminate its role as an endocrine organ. In addition, several studies have been carried out to reveal the role of EFT in the occurrence of pathogenic conditions. Specifically, in some studies, the lipid-storing depot characteristics of the EFT have been investigated, which mainly aimed to explain the secretion of cytokines and chemokines under pathogenic conditions as an inflammatory tissue [19]. Strong evidence shows that epicardial fat actively excretes many pro-inflammatory cytokines, such as tumour necrosis factor- $\alpha$ (TNF- $\alpha$ ), transforming growth factor- $\beta$ (TGF- $\beta$ ), interleukin-6 (IL-6), interleukin $\beta$ (IL-1 $\beta$ ), monocyte chemoattractant protein-1 (MCP-1) (a chemokine), and IL- $6 S R$ (interleukin 6 soluble receptor) [26]. It is stated in many studies that elevated epicardial fat tissue mass is observed in 
Table I. Clinical characteristics of the study population

\begin{tabular}{|c|c|c|c|c|}
\hline Variables & $\begin{array}{l}\text { All participants } \\
(n=1713)\end{array}$ & $\begin{array}{c}\text { Males } \\
(n=820)\end{array}$ & $\begin{array}{l}\text { Females } \\
(n=893)\end{array}$ & $P$-value \\
\hline Age [years] & $43.0(30.0-55.0)$ & $41.0(30.0-53.0)$ & $45.0(31.0-55.0)$ & $0.01^{\star}$ \\
\hline Tp-Te [ms] & $68.0(64.0-70.0)$ & $66.0(64.0-70.0)$ & $68.0(65.0-70.0)$ & $0.01^{*}$ \\
\hline QTmax [ms] & $363.0(355.0-374.0)$ & $361.0(353.0-371.0)$ & $365.0(356.0-375.0)$ & $<0.0001^{*}$ \\
\hline QTc [ms] & $399.8(383.8-420.0)$ & $394.2(380.1-412.5)$ & $405.9(388.8-424.9)$ & $<0.0001^{*}$ \\
\hline Tp-Te/QT ratio & $0.18(0.18-0.19)$ & $0.18(0.18-0.19)$ & $0.18(018-0.19)$ & 0.09 \\
\hline Tp-Te/QTc ratio\# & $0.17 \pm 0.015$ & $0.17 \pm 0.015$ & $0.17 \pm 0.014$ & 0.08 \\
\hline $\mathrm{Tp}-\mathrm{Te}(\mathrm{d})[\mathrm{ms}]$ & $15.0(10.0-20.0)$ & $15.0(10.0-20.0)$ & $15.0(10.0-20.0)$ & 0.1 \\
\hline $\mathrm{HR}$ & $74.0(70.0-80.0)$ & $71.0(67.0-79.0)$ & $75.0(70.0-80.0)$ & $<0.0001^{*}$ \\
\hline $\mathrm{PW}[\mathrm{mm}]$ & $8.0(7.0-9.0)$ & $8.0(7.4-9.0)$ & $8.0(7.0-8.0)$ & $<0.0001^{*}$ \\
\hline IVS [mm] & $8.0(7.0-9.0)$ & $8.0(8.0-9.0)$ & $8.0(7.0-9.0)$ & $<0.0001^{*}$ \\
\hline LVEDD $[\mathrm{mm}]$ & $44.0(42.0-46.0)$ & $45.0(43.0-47.0)$ & $43.0(41.0-45.0)$ & $<0.0001^{*}$ \\
\hline $\mathrm{EF}(\%)$ & $64.42(61.90-68.11)$ & $63.64(60.88-66.17)$ & $66.13(62.39-68.68)$ & $<0.0001^{*}$ \\
\hline FS (\%) & $34.88(33.33-37.50)$ & $34.42(32.60-36.31)$ & $36.17(33.33-38.09)$ & $<0.0001^{*}$ \\
\hline $\mathrm{BMI}\left[\mathrm{kg} / \mathrm{m}^{2}\right]$ & $26.42(24.91-28.58)$ & $26.33(24.83-28.37)$ & $26.57(24.97-28.80)$ & 0.10 \\
\hline $\mathrm{BSA}\left[\mathrm{m}^{2}\right]^{\#}$ & $1.87 \pm 0.17$ & $1.97 \pm 0.14$ & $1.77 \pm 0.15$ & $<0.0001^{*}$ \\
\hline LVEDV $[\mathrm{ml}]$ & $87.69(78.58-97.33)$ & $92.44(83.06-102.36)$ & $83.06(74.22-92.44)$ & $<0.0001^{*}$ \\
\hline LVEDV index $\left[\mathrm{ml} / \mathrm{m}^{2}\right]$ & $46.38(42.19-51.45)$ & $46.65(43.01-51.09)$ & $46.09(41.46-51.72)$ & 0.14 \\
\hline LV mass [g] & $106.87(92.06-132.82)$ & $117.90(100.60-142.70)$ & $97.33(85.07-122.53)$ & $<0.0001^{*}$ \\
\hline LV mass index $\left[\mathrm{g} / \mathrm{m}^{2}\right]$ & $57.49(49.85-69.59)$ & $59.89(51.82-71.81)$ & $55.40(48.58-67.24)$ & $<0.0001^{*}$ \\
\hline $\mathrm{HDL}-\mathrm{C}[\mathrm{mg} / \mathrm{dl}]$ & $46.0(41.0-52.0)$ & $43.0(39.0-47.0)$ & $50.0(45.0-56.0)$ & $<0.0001^{*}$ \\
\hline LDL-C [mg/dl] & $99.9(77.0-120.0)$ & $101.0(77.12-120.0)$ & $96.0(76.0-117.0)$ & $0.01^{*}$ \\
\hline Triglycerides [mg/dl] & $120.0(80.0-150.0)$ & $120.0(82.0-143.75)$ & $119.0(77.36-153.0)$ & 0.53 \\
\hline Fasting plasma glucose [mg/dl] & $91.0(84.0-100.3)$ & $92.0(85.0-102.0)$ & $90.4(82.6-100.0)$ & $0.01^{*}$ \\
\hline White blood cell $\left[\times 10^{3} / \mathrm{mm}^{3}\right]$ & $7.0(5.8-8.6)$ & $7.0(5.8-8.5)$ & $7.1(5.7-8.7)$ & 0.13 \\
\hline Haemoglobin [g/dl] & $14.0(13.2-15.0)$ & $14.3(13.5-15.0)$ & $13.6(13.0-14.6)$ & $<0.0001^{*}$ \\
\hline Haematocrit (\%) & $42.0(39.8-45.0)$ & $43.0(41.0-45.0)$ & $41.0(39.0-44.0)$ & $<0.0001^{*}$ \\
\hline Platelet $\left[\times 10^{3} / \mathrm{mm}^{3}\right]$ & $254.0(214.0-298.0)$ & $249.5(210.0-289.0)$ & $260.0(217.0-301.5)$ & $<0.0001^{*}$ \\
\hline Sodium $[\mathrm{mmol} / \mathrm{l}]$ & $139.0(137.0-142.0)$ & $139.0(137.0-142.0)$ & $140.0(138.0-142.0)$ & 0.13 \\
\hline Potassium $[\mathrm{mEq} / \mathrm{l}]$ & $4.2(4.0-4.6)$ & $4.3(4.0-4.6)$ & $4.2(4.0-4.6)$ & 0.10 \\
\hline Calcium [mg/dl] & $9.2(8.9-9.6)$ & $9.2(8.9-9.6)$ & $9.2(9.0-9.6)$ & 0.78 \\
\hline Creatinine $[\mathrm{mg} / \mathrm{dl}]$ & $0.69(0.54-0.81)$ & $0.72(0.60-0.87)$ & $0.62(0.50-0.75)$ & $<0.0001^{*}$ \\
\hline Urea [mg/dl] & $30.0(25.0-36.0)$ & $31.0(26.0-38.0)$ & $30.0(24.0-36.0)$ & $<0.0001^{*}$ \\
\hline EFTtd $[\mathrm{mm}]$ & $2.5(1.8-3.2)$ & $2.2(1.7-3.0)$ & $2.6(2.0-3.5)$ & $<0.0001^{*}$ \\
\hline EFTts [mm] & $5.0(3.4-6.0)$ & $5.0(3.5-5.5)$ & $5.0(3.2-6.0)$ & $0.001^{*}$ \\
\hline
\end{tabular}

$B M I$ - body mass index, BSA - body surface area, EF - ejection fraction, HR - heart rate, $L V$ - left ventricle, LVEDD - left ventricular end-diastolic diameter, LVESD - left ventricular end-systolic diameter, PW - posterior wall, IVS - interventricular septum, LVEDV - left ventricular end-diastolic volume, HDL-C - high-density lipoprotein-cholesterol, LDL-C - low-density lipoprotein-cholesterol, EFTtd -

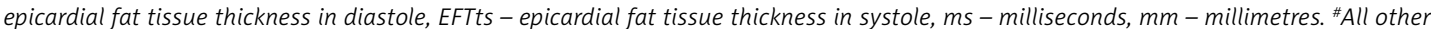
continuous values except Tp-Te/QTc ratio and BSA $\left(\mathrm{m}^{2}\right)$ did not have normal distribution, and the Kolmogorov-Smirnov test was used to

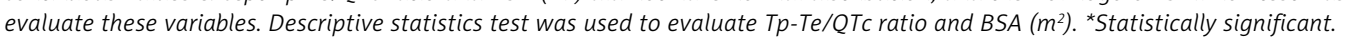


M. Yılmaz, H. Kayançiçek, N. Gözel, E. Kurtoğlu, Ö. Seçen, P. Öner, Y. Çekici, M. Nail Bilen, S. Demirkıran, Ö. Uku

Table II. Some clinical characteristics of the study population in terms of BMI

\begin{tabular}{|c|c|c|c|c|}
\hline Parameter & $\begin{array}{c}\mathrm{BMI}<25 \mathrm{~kg} / \mathrm{m}^{2} \\
(n=450)\end{array}$ & $\begin{array}{c}25 \mathrm{~kg} / \mathrm{m}^{2}<\mathrm{BMI} \\
<30 \mathrm{~kg} / \mathrm{m}^{2} \\
(n=1048)\end{array}$ & $\begin{array}{c}30 \mathrm{~kg} / \mathrm{m}^{2}<\mathrm{BMI} \\
(n=215)\end{array}$ & $P$-value \\
\hline Age [years] & $30.0(21.0-45.0)$ & $46.0(33.0-56.0)$ & $48.0(35.0-58.0)$ & $<0.0001,0.55^{x}$ \\
\hline Tp-Te [msn] & $63.0(60.0-66.0)$ & $68.0(66.0-70.0)$ & $72.0(68.0-75.0)$ & $<0.0001$ \\
\hline QTmax [ms] & $356.0(345.0-365.0)$ & $365.0(358.0-375.0)$ & $375.0(366.0-382.0)$ & $<0.0001$ \\
\hline QTc [ms] & $394.5(378.8-415.3)$ & $399.8(384.5-419.2)$ & $413.6(394.3-435.6)$ & $<0.0001$ \\
\hline Tp-Te/QT ratio & $0.18(0.17-0.18)$ & $0.18(0.18-0.19)$ & $0.19(0.18-0.20)$ & $<0.0001$ \\
\hline Tp-Te/QTc ratio\# & $0.16 \pm 0.014$ & $0.17 \pm 0.014$ & $0.17 \pm 0.015$ & $<0.0001,0.001^{x}$ \\
\hline $\mathrm{Tp}-\mathrm{Te}(\mathrm{d})[\mathrm{ms}]$ & $10.0(10.0-15.0)$ & $16.0(10.0-20.0)$ & $20.0(12.0-25.0)$ & $<0.0001$ \\
\hline EFTtd [mm] & $1.5(1.0-2.2)$ & $2.6(2.0-3.2)$ & $3.5(3.0-4.5)$ & $<0.0001$ \\
\hline EFTts [mm] & $3.4(3.0-5.0)$ & $5.0(4.0-6.0)$ & $6.1(5.2-7.0)$ & $<0.0001$ \\
\hline BMI $\left[\mathrm{kg} / \mathrm{m}^{2}\right]$ & $23.18(21.77-24.33)$ & $26.87(26.02-28.13)$ & $32.40(31.02-34.89)$ & $<0.0001$ \\
\hline BSA $\left[\mathrm{m}^{2}\right]^{\#}$ & $1.74 \pm 0.15$ & $1.88 \pm 0.14$ & $2.06 \pm 0.18$ & $<0.0001$ \\
\hline LVEDV [ml] & $78.58(70.0-87.69)$ & $92.44(78.58-97.33)$ & $92.44(83.06-107.52)$ & $<0.0001$ \\
\hline LVEDV index $\left[\mathrm{ml} / \mathrm{m}^{2}\right]$ & $44.72(41.42-49.08)$ & $47.30(43.18-52.35)$ & $45.72(40.90-51.30)$ & $\begin{array}{c}<0.0001,0.11^{*} \\
0.32^{x}\end{array}$ \\
\hline LV mass $[\mathrm{g}]$ & $93.04(81.68-105.07)$ & $113.88(96.78-137.72)$ & $123.29(101.29-158.82)$ & $<0.0001$ \\
\hline LV mass index $\left[\mathrm{g} / \mathrm{m}^{2}\right]$ & $52.24(47.62-59.38)$ & $60.42(51.45-71.91)$ & $60.36(50.47-73.88)$ & $<0.0001,1.0^{x}$ \\
\hline
\end{tabular}

Table III. Pearson's correlation analysis between EFTt and baseline characteristics, echocardiography, and some laboratory measurements

\begin{tabular}{|lcccc|}
\hline Parameter & \multicolumn{2}{c}{ EFTtd } & \multicolumn{2}{c|}{ EFTts } \\
\cline { 2 - 5 } & $r$ & $P$-value & $r$ & $P$-value \\
\hline Age & 0.580 & $<0.0001$ & 0.581 & $<0.0001$ \\
\hline Fasting plasma glucose [mg/dl] & 0.127 & $<0.0001$ & 0.134 & $<0.0001$ \\
\hline LDL-C [mg/dl] & 0.135 & $<0.0001$ & 0.135 & $<0.0001$ \\
\hline Triglycerides [mg/dl] & 0.330 & $<0.0001$ & 0.309 & $<0.0001$ \\
\hline HDL-C [mg/dl] & -0.142 & $<0.0001$ & -0.170 & $<0.0001$ \\
\hline Tp-Te $[\mathrm{msn}]$ & 0.564 & $<0.0001$ & 0.566 & $<0.0001$ \\
\hline Tp-Te/QT ratio & 0.377 & $<0.0001$ & 0.390 & $<0.0001$ \\
\hline Tp-Te $(\mathrm{d})[\mathrm{msn}]$ & 0.496 & $<0.0001$ & 0.492 & $<0.0001$ \\
\hline BMI [kg/m $\left.{ }^{2}\right]$ & 0.500 & $<0.0001$ & 0.518 & $<0.0001$ \\
\hline BSA [m $\left.{ }^{2}\right]$ & 0.213 & $<0.0001$ & 0.243 & $<0.0001$ \\
\hline LV mass [g] & 0.473 & $<0.0001$ & 0.469 & $<0.0001$ \\
\hline LV mass index [g/m²] & 0.452 & $<0.0001$ & 0.439 & $<0.0001$ \\
\hline LVEDV [ml] & 0.434 & $<0.0001$ & 0.423 & $<0.0001$ \\
\hline LVEDV index [ml/m²] & 0.381 & $<0.0001$ & 0.354 & $<0.0001$ \\
\hline
\end{tabular}

LDL-C - low-density lipoprotein-cholesterol, HDL-C - high-density lipoprotein-cholesterol, BMI - body mass index, BSA - body surface area, LV mass - left ventricle mass. 

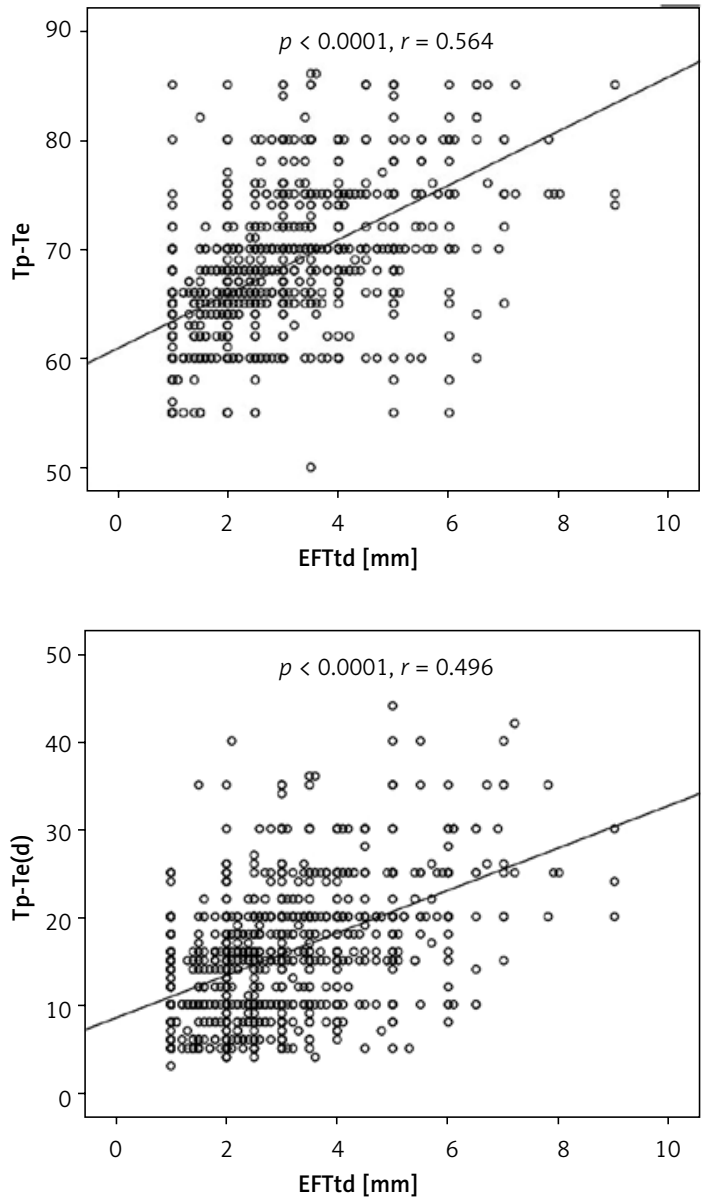

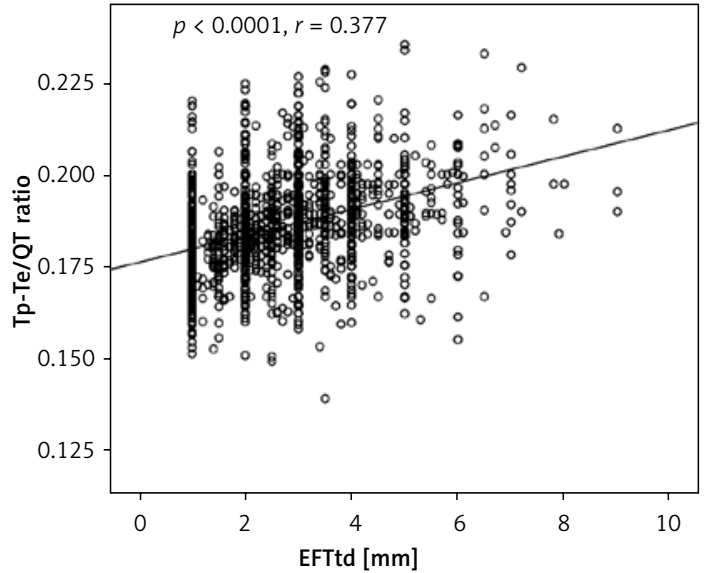

Figure 7. The correlations between Tp-Te interval, $\mathrm{Tp}$-Te/QT ratio, Tp-Te interval dispersion $\mathrm{Tp}-\mathrm{Te}(\mathrm{d})$, and EFTt in diastole (EFTtd)

Table IV. Multivariate linear regression analysis results for $\mathrm{Tp}$-Te

\begin{tabular}{|lcccc|}
\hline Parameter & $\beta$ & Standard error & $95 \%$ confidence interval & $P$-value \\
\hline EFTtd & 0.163 & 0.161 & $-0.152-0.478$ & 0.31 \\
\hline EFTts & 0.224 & 0.127 & $-0.024-0.473$ & 0.08 \\
\hline BMI [kg/m²] & 0.543 & 0.037 & $0.471-0.615$ & $<0.0001^{*}$ \\
\hline BSA [m²] & -1.599 & 3.858 & $-9.165-5.968$ & 0.68 \\
\hline LV mass [g] & 0.232 & 0.058 & $0.117-0.347$ & $<0.0001^{*}$ \\
\hline LV mass index [g/m²] & -0.144 & 0.120 & $0.379-0.091$ & 0.23 \\
\hline LVEDV [ml] & -0.390 & 0.135 & $-0.656-0.125$ & $0.05^{*}$ \\
\hline LVEDV index [ml/m²] & 0.346 & 0.220 & $-0.086-0.778$ & 0.11 \\
\hline Age & 0.067 & 0.007 & $0.052-0.082$ & $<0.0001^{*}$ \\
\hline Fasting plasma glucose [mg/dl] & 0.008 & 0.007 & $-0.005-0.021$ & 0.23 \\
\hline LDL-C [mg/dl] & -0.004 & 0.002 & $-0.008-0.000$ & 0.07 \\
\hline Triglycerides [mg/dl] & 0.001 & 0.001 & $0.000-0.004$ & 0.24 \\
\hline HDL-C [mg/dl] & 0.006 & 0.009 & $-0.011-0.024$ & 0.46 \\
\hline
\end{tabular}

$R^{2}=0.66, p<0.0001 .{ }^{*}$ Statistically significant.

patients with paroxysmal atrial fibrillation in comparison to the controls, and even higher epicardial fat mass is detected in patients with permanent
AF when compared to patients with paroxysmal atrial fibrillation [27, 28]. Another study proved that the inflammatory markers are more eminent- 
Table V. Multivariate linear regression analysis results for $\mathrm{Tp}-\mathrm{Te} / \mathrm{QT}$ ratio

\begin{tabular}{|c|c|c|c|c|}
\hline Paramter & $\beta$ & Standard error & 95\% confidence interval & $P$-value \\
\hline EFTtd & 0.000 & 0.000 & $-0.001-0.000$ & 0.33 \\
\hline EFTts & 0.001 & 0.000 & $0.000-0.002$ & 0.06 \\
\hline $\mathrm{BMI}\left[\mathrm{kg} / \mathrm{m}^{2}\right]$ & 0.001 & 0.000 & $0.001-0.001$ & $<0.0001^{*}$ \\
\hline $\mathrm{BSA}\left[\mathrm{m}^{2}\right]$ & 0.002 & 0.011 & $-0.020-0.024$ & 0.83 \\
\hline LV mass $[\mathrm{g}]$ & 0.000 & 0.000 & $0.000-0.001$ & $0.005^{*}$ \\
\hline LV mass index $\left[\mathrm{g} / \mathrm{m}^{2}\right]$ & 0.000 & 0.000 & $-0.001-0.000$ & 0.11 \\
\hline LVEDV [ml] & 0.000 & 0.000 & $-0.002-0.000$ & $0.02^{*}$ \\
\hline LVEDV index $\left[\mathrm{ml} / \mathrm{m}^{2}\right]$ & 0.001 & 0.001 & $0.000-0.003$ & $0.04^{*}$ \\
\hline Age & $-3.651 \times 10^{-5}$ & 0.000 & $0.000-0.000$ & $0.009^{*}$ \\
\hline Fasting plasma glucose [mg/dl] & $1.154 \times 10^{-5}$ & 0.000 & $0.000-0.000$ & 0.54 \\
\hline LDL-C [mg/dl] & $-2.032 \times 10^{-5}$ & 0.000 & $0.000-0.000$ & $0.001^{*}$ \\
\hline Triglycerides [mg/dl] & $1.742 \times 10^{-6}$ & 0.000 & $0.000-0.000$ & 0.61 \\
\hline $\mathrm{HDL}-\mathrm{C}[\mathrm{mg} / \mathrm{dl}]$ & $-2.867 \times 10^{-5}$ & 0.000 & $0.000-0.000$ & 0.26 \\
\hline
\end{tabular}

$R^{2}=0.382, p<0.0001$. ${ }^{*}$ Statistically significant.

ly related to the periatrial epicardial fat volume than the thickness of the EFT. In this respect, the local influence of EFT exhibits a vital role in AF pathogenesis [29].

Detection and quantification of EFT require a variety of useful imaging techniques, including two-dimensional (2D) echocardiography, non-contrast computed tomography (CT), and magnetic resonance imaging (MRI) [30,31]. The EFTt can be evaluated and visualised by a two-dimensional echocardiographic method, which is noninvasive, objective, reliable, easily obtainable, and less expensive than the gold standard MRI and CT. The identification of the EFTt is straightforward, in the sense that it can be easily discriminated by determining the echo-free space between the pericardial visceral segment and the myocardial outer wall. Also, the EFTt is evaluated between the epicardial surface and the parietal pericardium in at least two locations on the right ventricular free wall [18]. Determination of the best cardiac cycle stage for taking measurements of echocardiography is a controversial issue. Some recommend that measurement should be taken during systole to prohibit potential deformation from epicardial fat compression during diastole, while others suggest measurement in diastole is best in order to match up with other imaging methods (CT and MRI) [30, 32, 33].

Abnormality observed in repolarisation of the ventricle has been considered as a significant indicator of ventricular arrhythmogenesis [34]. One way to detect such abnormalities is by marking the highest point and final point of the $T$ wave on
ECG, which is a marker of total TDR (transmural, apicobasal, and global). When an increase is observed in the interval of Tp-Te, it can be concluded that the risk of ventricular tachyarrhythmia and cardiovascular mortality will increase [35-38]. Tp-Te indicates the maximum dispersion of repolarisation. On the other hand, the variety of TDR within various zones of the ventricular myocardium can be detected by Tp-Te (d) [11]. Finally, focusing only on the intervals of Tp-Te or QT is generally not adequate to draw accurate conclusions. Instead, Tp-Te/QT ratio can be utilised to gain a more sensitive index of arrhythmogenesis, so that this ratio remains stable even if the body weight or heart rate values varies [5]. Recently, these three electrocardiographic parameters have come to be considered the most useful markers as indirect indexes of ventricular repolarisation for arrhythmic vulnerability [11,39].

It is stated that there is a strong relation between the increased fibrinogen, C-reactive protein, cholesterol levels, and physical inactivity and high BMI values [40]. In another study, it was proven that there is an association between the ventricular tachyarrhythmias and prolonged QT durations. In addition, when obesity exists, an increase in QT and QTd parameters is observed [41]. In some people who are overweight or obese, even without heart disease, cardiomyocytes can demonstrate some abnormities in their electrophysiological features. These are characterised by a lengthening of the action potential [39]. Mora et al. stated that a considerable association exists between cardiac sympathetic nervous activity and repolarisation 
abnormities in people who are overweight or obese [40]. On the other hand, Acar et al. stated that ventricular repolarisation markers derived from ECG are associated with systemic inflammation [42] However, inflammatory biomarkers of plasma might not sufficiently represent regional tissue inflammation. EFT is a resource of many inflammatory biological agents. In addition, Mazurek et al. reported that serum HDL levels and the epicardial expression of IL-6SR are inversely correlated [26].

It has been reported that, with aging, fibrosis effects start to occur in myocardium, which yields an increase in ventricular repolarisation heterogeneity [43]. Another explanation for the increase in TDR with age is distortion of the balance between sympathetic and parasympathetic tone in favour of the sympathetic tone. In old age, this balance changes in favour of the sympathetic activity considerably [44]. High sympathetic activity can generate several changes in myocardial membrane characteristics, which cause to early after depolarisations and an increase in TDR [45-47]. Our study shows a relationship between age, BMI, BSA, and EFTt (Table III). In other words, increased BMI, BSA, and advancing age lead to increased EFTt. Although the details of the aforementioned relation are still an unexplored issue, aging, BMI, and BSA may lead to an increase in sympathetic activity in these subjects, and this situation may contribute to the increased ventricular repolarisation heterogeneity as well.

Multivariate linear regression analysis results revealed that age, BMI $\left(\mathrm{kg} / \mathrm{m}^{2}\right)$, LV mass $(\mathrm{g})$, LVEDV $(\mathrm{ml})$, and LVEDV index $\left(\mathrm{ml} / \mathrm{m}^{2}\right)$ are independently related to $\mathrm{Tp}-\mathrm{Te}$ interval and $\mathrm{Tp}-\mathrm{Te} / \mathrm{QT}$ ratio. However, such a relationship could not be detected between EFTd and EFTs and Tp-Te and $\mathrm{Tp}$-Te/QT ratio (Tables IV, V). These data reveal that age, BMI $\left(\mathrm{kg} / \mathrm{m}^{2}\right)$, LV mass $(\mathrm{g})$, LVEDV $(\mathrm{ml})$, and LVEDV index $\left(\mathrm{ml} / \mathrm{m}^{2}\right)$ are independent predictors for increased ventricular repolarisation heterogeneity, but EFTtd and EFTts are not predictors for TDR. All these results show that EFTtd and EFTts values increase with age, BMI $\left(\mathrm{kg} / \mathrm{m}^{2}\right)$, LV mass (g), LVEDV $(\mathrm{ml})$, and LVEDV index $\left(\mathrm{ml} / \mathrm{m}^{2}\right)$ in healthy individuals. This increase correlates positively with TDR parameters and indicates that all of the data mentioned are responsible for increased ventricular repolarization heterogeneity.

In light of former research outcomes, in this study we considered that there may be a direct relationship between EFTt and dysrhythmia [2629, 39-42]. We hypothesised that EFT may lead to structural and electrical remodelling of the myocardium and thus may contribute to the formation of ventricular arrhythmias, because of its closeness to the myocardium and its feeding by the same coronary arteries.

The present study included 1713 healthy participants, 1048 of whom were overweight $\left(25 \mathrm{~kg} / \mathrm{m}^{2}\right.$
$\left.<\mathrm{BMI}<30 \mathrm{~kg} / \mathrm{m}^{2}\right)$ and 215 were obese $\left(30 \mathrm{~kg} / \mathrm{m}^{2}\right.$ < BMI) (Table II). All factors and diseases that could form the basis for ventricular arrhythmia were excluded, and only factors that would show the impact of EFTt on ventricular arrhythmia predisposition were investigated. We observed that the TDR parameters increased in direct proportion to the EFT thickness and that, at the same time, aging, BSA, BMI, LV volume, LV volume index, LV mass, LV mass index, fasting plasma glucose, triglycerides, and LDL had a positive correlation with the EFTt. Conversely, the HDL levels had a negative correlation with the EFTt (Table III). Inflammation can be a reasonable explanation for the positive correlation between the EFTt and TDR parameters (Tp-Te interval Tp-Te(d), Tp-Te/QT ratio, Tp-Te/QTc ratio) that were observed in this research study. In addition, the risk of ventricular repolarisation inhomogeneity elevates in parallel with the enhanced sympathetic nervous system activity. In this study, repolarisation abnormalities were observed in older, overweight, and obese participants, and it can also show an incidental coexistence with the relative increase in the EFTt, which occurs due to different causes in the same participants. There does not seem to be a clear-cut answer to this issue. However, in light of the available findings, we can say that there is a moderate correlation between the increase in the EFTt measured echocardiographically and the increase in the ventricular repolarisation heterogeneity measured electrocardiographically.

In conclusion, as of today, the details of the relationship between the ventricular arrhythmias and increased EFTt are not clearly explained. The results of the study demonstrated that the EFT may has a direct effect on the ventricular repolarisation inhomogeneity, and these results imply that an increase in EFTt may lead to an augmentation in the inhomogeneity of ventricular repolarisation via myocardial electrical reconstruction.

Today, QT distance and QT dispersion are known to increase in parallel with obesity. The study included 215 obese participants, and this may have partially affected the correlation analyses performed between TDR parameters and EFTt.

\section{Conflict of interest}

The authors declare no conflict of interest.

\section{References}

1. Nagy E, Jermendy AL, Merkely B, et al. Clinical importance of epicardial adipose tissue. Arch Med Sci 2017; 13: 864-74.

2. Patel VB, Shah S, Verma S, et al. Epicardial adipose tissue as a metabolic transducer: role in heart failure and coronary artery disease. Heart Fail Rev 2017; 22: 889902. 
3. Kors JA, van Eck HJR, van Herpen G. The meaning of the Tp-Te interval and its diagnostic value. J Electrocardiol 2008; 41: 575-80.

4. Antzelevitch C, Sicouri S, Di Diego JM, et al. Does TpeakTend provide an index of transmural dispersion of repolarization? Heart Rhythm 2007; 4: 1114-6

5. Gupta P, Patel C, Patel H, et al. Tp-e/QT ratio as an index of arrhythmogenesis. J Electrocardiol 2008; 41: 567-74.

6. Zhao X, Xie Z, Chu Y, et al. Association between Tp-e/ QT ratio and prognosis in patients undergoing primary percutaneous coronary intervention for ST-segment elevation myocardial infarction. Clin Cardiol 2012; 35: 559-64.

7. Balcıoğlu AS, Erdoğan T, Şatıroğlu Ö. The relationship between epicardial adipose tissue and P wave and QT dispersions. Turk Kardiyol Dern Ars 2015; 43: 621-9.

8. lacobellis G, Corradi D, Sharma AM. Epicardial adipose tissue: anatomic, biomolecular and clinical relationships with the heart. Nat Rev Pract Cardiovasc Med 2005; 2: 536-43.

9. Kaplan O, Kurtoglu E, Nar G, et al. Evaluation of electrocardiographic T-peak to T-end interval in subjects with increased epicardial fat tissue thickness. Arq Brasil Cardiol 2015; 105: 566-72.

10. Burns E. ECG Basıcs: Waves, Intervals, Segments and Clinical Interpretation. [Accessed May 20, 2018]. Available from: https://lifeinthefastlane.coms ECG Library.

11. Hevia JC, Antzelevitch C, Bárzaga FT, et al. Tpeak-Tend and Tpeak-Tend dispersion as risk factors for ventricular tachycardia/ventricular fibrillation in patients with the Brugada syndrome. J Am Coll Cardiol 2006; 47: 1828-34.

12. Icli A, Kayrak M, Akilli H, et al. Prognostic value of TpeakTend interval in patients with acute pulmonary embolism. BMC Cardiovasc Disord 2015; 15: 99.

13. Lang RM, Bierig M, Devereux RB, et al. Recommendations for chamber quantification. Eur J Echocardiogr 2006; 7: 79-108.

14. lacobellis G, Ribaudo MC, Assael F, et al. Echocardiographic epicardial adipose tissue is related to anthropometric and clinical parameters of metabolic syndrome: a new indicator of cardiovascular risk. J Clin Endocrinol Metab 2003; 88: 5163-8.

15. Lang RM, Bierig M, Devereux RB, et al. Recommendations for chamber quantification: a report from the American Society of Echocardiography's Guidelines and Standards Committee and the Chamber Quantification Writing Group, developed in conjunction with the European Association of Echocardiography, a branch of the European Society of Cardiology. J Am Soc Echocardiogr 2005; 18: 1440-63.

16. Hudsmith LE, Petersen SE, Francis JM, et al. Normal human left and right ventricular and left atrial dimensions using steady state free precession magnetic resonance imaging. J Cardiovasc Magn Reson 2005; 7: 775-82.

17. Bruce R, Blackmon J, Jones J, et al. Exercising testing in adult normal subjects and cardiac patients. Ann Noninvasive Electrocardiol 2004; 9: 291-303.

18. Wu Y, Zhang A, Hamilton DJ, et al. Epicardial fat in the maintenance of cardiovascular health. Methodist DeBakey Cardiovasc J 2017; 13: 20-24.

19. Cherian S, Lopaschuk GD, Carvalho E. Cellular cross-talk between epicardial adipose tissue and myocardium in relation to the pathogenesis of cardiovascular disease. Am J Physiol Endocrinol Metab 2012; 303: E937-49.

20. Patel VB, Mori J, McLean BA, et al. ACE2 deficiency worsens epicardial adipose tissue inflammation and cardiac dysfunction in response to diet-induced obesity. Diabetes 2016; 65: 85-95.

21. Toczyłowski K, Gruca M, Baranowski M. Epicardial adipose tissue and its role in cardiac physiology and disease. Postepy Hig Med Dosw 2013; 67: 584-93.

22. Çetin M, Erdogan T, Kocaman SA, et al. Increased epicardial adipose tissue in patients with isolated coronary artery ectasia. Int Med 2012; 51: 833-8.

23. Eroglu S., Sade L.E., Yildirir A., et al. Epicardial adipose tissue thickness by echocardiography is a marker for the presence and severity of coronary artery disease. Nutrition Metabolism Cardiovasc Dis 2009; 19: 211-7.

24. Erdogan T, Canga A, Kocaman SA, et al. Increased epicardial adipose tissue in patients with slow coronary flow phenomenon. Kadiol Pol 2012; 70: 903-9.

25. Sacks HS, Fain JN. Human epicardial adipose tissue: a review. Am Heart J 2007; 153: 907-17.

26. Mazurek T, Zhang L, Zalewski A, et al. Human epicardial adipose tissue is a source of inflammatory mediators. Circulation 2003; 108: 2460-6.

27. Acet $\mathrm{H}$, Ertaş F, Akıl MA, et al. New inflammatory predictors for non-valvular atrial fibrillation: echocardiographic epicardial fat thickness and neutrophil to lymphocyte ratio. Int J Cardiovasc Imaging 2014; 30: 81-9.

28. lacobellis G, Zaki M, Garcia D, et al. Epicardial fat in atrial fibrillation and heart failure. Horm Metab Res 2014; 46: 587-90.

29. Girerd N, Scridon A, Bessière F, et al. Periatrial epicardial fat is associated with markers of endothelial dysfunction in patients with atrial fibrillation. PLoS One 2013; 8: e77167

30. Nelson AJ, Worthley MI, Psaltis PJ, et al. Validation of cardiovascular magnetic resonance assessment of pericardial adipose tissue volume. J Cardiovasc Magn Reson 2009; $11: 15$.

31. Iacobellis G, Willens HJ. Echocardiographic epicardial fat: a review of research and clinical applications. J Am Soc Echocardiogr 2009; 22: 1311-9.

32. lacobellis G. Local and systemic effects of the multifaceted epicardial adipose tissue depot. Nat Rev Endocrinol 2015; 11: 363-71.

33. Mookadam F, Goel R, Alharthi MS, et al. Epicardial fat and its association with cardiovascular risk: a cross-sectional observational study. Heart Views 2010; 11: 103-8.

34. Salles G, Xavier S, Sousa A, et al. Prognostic value of QT interval parameters for mortality risk stratification in Chagas' disease: results of a long-term follow-up study. Circulation 2003; 108: 305-12.

35. Tenekecioglu E, Karaagac K, Yontar OC, et al. Evaluation of Tp-Te Interval and Tp-Te/QT ratio in patients with coronary slow flow Tp-Te/QT ratio and coronary slow flow. Eurasian J Med 2015; 47: 104-8.

36. Smetana P, Schmidt A, Zabel M, et al. Assessment of repolarization heterogeneity for prediction of mortality in cardiovascular disease: peak to the end of the T wave interval and nondipolar repolarization components. J Electrocardiol 2011; 44: 301-8.

37. Erikssen G, Liestøl K, Gullestad L, et al. The terminal part of the QT interval (T peak to $T$ end): a predictor of mortality after acute myocardial infarction. Ann Noninvasive Electrocardiol 2012; 17: 85-94.

38. Karaagac K, Tenekecioglu E, Yontar OC, et al. Effect of non-dipper and dipper blood pressure patterns on Tp-Te interval and Tp-Te/QT ratio in patients with metabolic syndrome. Int J Clin Exp Med 2014; 7: 1397-403.

39. Braschi A, Abrignani MG, Francavilla VC, et al. Novel electrocardiographic parameters of altered repolariza- 
tion in uncomplicated overweight and obesity. Obesity 2011; 19: 875-81.

40. Mora S, Lee IM, Buring JE, et al. Association of physical activity and body mass index with novel and traditional cardiovascular biomarkers in women. JAMA 2006; 295 : 1412-9.

41. Esposito K, Nicoletti G, Marzano S, et al. Autonomic dysfunction associates with prolongation of QT intervals and blunted night $\mathrm{BP}$ in obese women with visceral obesity. J Endocrinol Invest 2002; 25: RC32-5.

42. Acar G, Yorgun H, Inci MF, et al. Evaluation of Tp-e interval and Tp-e/QT ratio in patients with ankylosing spondylitis. Modern Rheumatol 2014; 24: 327-30.

43. Reardon M, Malik M. QT interval change with age in an overtly healthy older population. Clin Cardiol 1996; 19: 949-52.

44. Pfeifer MA, Weinberg CR, Cook D, et al. Differential changes of autonomic nervous system function with age in man. Am J Med 1983; 75: 249-58.

45. Zipes DP. The long QT interval syndrome. A Rosetta stone for sympathetic related ventricular tachyarrhythmias. Circulation 1991; 84: 1414-9.

46. Ben-David J, Zipes DP. Differential response to right and left ansae subclaviae stimulation of early afterdepolarizations and ventricular tachycardia induced by cesium in dogs. Circulation 1988; 78: 1241-50.

47. Vincent GM, Timothy KW, Leppert M, et al. The spectrum of symptoms and QT intervals in carriers of the gene for the long-QT syndrome. N Engl J Med 1992; 327: 846-52. 